\title{
Belgeo
}

Revue belge de géographie

\section{La périurbanisation résidentielle en Belgique à la lumière des contextes suisse et danois: enracinement, dynamiques centrifuges et régulations collectives}

Residential peri-urbanisation in Belgium, Switzerland and Denmark: implantation, centrifugal dynamics and collective regulations

Jean-Marie Halleux, Laurent Brück et Nicolas Mairy

\section{(2) OpenEdition}

\section{Journals}

Édition électronique

URL : http://journals.openedition.org/belgeo/16086

DOI : 10.4000/belgeo.16086

ISSN : 2294-9135

Éditeur :

National Committee of Geography of Belgium, Société Royale Belge de Géographie

Édition imprimée

Date de publication : 30 décembre 2002

Pagination : 333-354

ISSN : 1377-2368

Référence électronique

Jean-Marie Halleux, Laurent Brück et Nicolas Mairy, «La périurbanisation résidentielle en Belgique à la lumière des contextes suisse et danois: enracinement, dynamiques centrifuges et régulations collectives », Belgeo [En ligne], 4 | 2002, mis en ligne le 31 décembre 2002, consulté le 11 février 2021. URL : http://journals.openedition.org/belgeo/16086 ; DOI : https://doi.org/10.4000/belgeo.16086

Ce document a été généré automatiquement le 11 février 2021.

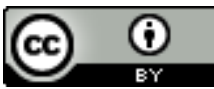

Belgeo est mis à disposition selon les termes de la licence Creative Commons Attribution 4.0 International. 


\title{
La périurbanisation résidentielle en Belgique à la lumière des contextes suisse et danois: enracinement, dynamiques centrifuges et régulations collectives
}

\author{
Residential peri-urbanisation in Belgium, Switzerland and Denmark:
} implantation, centrifugal dynamics and collective regulations

Jean-Marie Halleux, Laurent Brück et Nicolas Mairy

En Belgique, la géographie des périphéries urbaines a connu de très profondes mutations lors des dernières décennies. De nombreuses activités économiques s'y sont implantées et des formes d'habitat desserré et dispersé y ont colonisé de très vastes territoires. En d'autres termes, la périurbanisation, ce processus de déploiement spatial des villes, s'y est très largement imposée. Rendu possible par la banalisation de la mobilité voiture et alimenté par de multiples dynamiques centrifuges, le phénomène périurbain belge, désormais, inquiète. En dépit de mises en garde relativement anciennes (Sporck et Van der Haegen, 1985, pp. 263-265), les réponses à l'extension inorganisée des périphéries n'ont été que partielles et il est à présent manifeste que l'émergence de formes urbaines très largement façonnées par la conduite automobile porte en son sein les germes de sérieuses atteintes à la durabilité. De fait, des études de plus en plus nombreuses et étayées ${ }^{1}$ concluent sur les surcoûts collectifs de long terme qu'entraîne une gestion territoriale inappropriée de la banalisation de l'automobile: consommations importantes d'espaces naturels et agricoles, surcoûts publics en équipements de terrains et en constructions d'infrastructures, multiplication des friches urbaines, dilatation potentielle des ségrégations socio-spatiales et forte croissance des externalités négatives générées par le trafic routier, tant au niveau local qu'à l'échelle planétaire. 
2 Les dynamiques dispersives qui alimentent les développements périurbains belges sont naturellement communes à de nombreux pays (Dubois-Taine, 2002). Ainsi, dans de nombreux pays, on observe la dynamique technique de l'amélioration des réseaux de transports et la dynamique économique de l'appropriation de biens de consommation matériels tels que l'automobile ou la villa périurbaine. Dans de nombreux pays, on observe également les dynamiques plus sociales du recentrage sur la sphère familiale et la valorisation d'un certain isolement rural, au détriment de la sphère collective. Bien que présentes partout en Europe, ces forces centrifuges n'aboutissent pas nécessairement à des résultats identiques. A chaque contexte correspondent de complexes mécanismes systémiques où jouent simultanément le poids des structures historiques, les préférences des populations et les mécanismes de régulation des politiques publiques. In fine, à cette diversité dans la production de l'espace urbain vont correspondre une diversité dans l'endiguement de l'étalement et, concomitamment, une diversité dans le degré d'accrochage entre les évolutions urbaines et les options stratégiques du développement durable.

3 Le concept de périurbanisation n'a, malheureusement, pas encore été internationalement normalisé. Il reste donc délicat d'établir des comparaisons internationales quant à l'intensité du déploiement spatial des villes. Toutefois, au regard de nombreux pays proches, la Belgique semble marquée par une périurbanisation à la fois plus soutenue et plus diffuse (De Lannoy et De Corte, 2000, p. 191). En attestent notamment la pression sur les paysages (Antrop, 2000, pp. 21-22), la faible densité des agglomérations (Vandermotten et al., 1999), la multiplication des polarités commerciales (Mérenne-Schoumaker, 1998) ou encore la délocalisation périphérique de l'emploi (Colard et Vandermotten, 1996). Le présent article se propose d'étudier les causes de l'intense périurbanisation résidentielle qui affecte le territoire belge, cela à la lumière d'une comparaison internationale. Nous nous intéresserons ici plus particulièrement à la Suisse et au Danemark, deux nations réputées pour avoir mis sur pied des politiques relativement efficaces de lutte contre l'étalement urbain et fréquemment citées en exemple dans la littérature scientifique et technique pour leur capacité à inscrire l'évolution des villes dans les principes du développement durable (Brück et al., 2001b). Préciser cette comparaison entre Belgique, Suisse et Danemark va maintenant nous conduire à appréhender les trois thèmes de la politique foncière, de la production immobilière résidentielle et de la mobilité voiture.

\section{L'exploitation des gisements fonciers et la dynamique centrifuge de la rente d'urbanisation}

Physiquement, le déploiement périphérique de l'urbanisation nécessite l'exploitation d'importants gisements fonciers. Différentes conditions sont nécessaires à cette exploitation urbaine des terres et nous savons déjà qu'une première nécessité correspond au gain d'accessibilité induit par le relâchement des contraintes de transport. En effet, c'est l'accessibilité du terrain, elle-même déterminée par les performances des réseaux de transport, qui détermine si une localisation intéresse les opérateurs - promoteurs ou particuliers - qui produisent l'urbanisation (Halleux, 2001a). Pour rendre compte de l'exploitation des gisements fonciers, il faut envisager une seconde limitation potentielle, celle induite par la politique foncière. Dans le contexte belge, à la différence des situations danoise et suisse, on observe que cette 
possible régulation collective de la gestion publique des sols n'a que très partiellement permis d'endiguer l'extension périurbaine. Nous proposons maintenant de définir les raisons essentielles de cette situation qui, comme nous le verrons, entremêlent deux facteurs culturels: la prégnance du droit de propriété et la faible conscientisation aux enjeux de la planification.

\section{La gestion foncière en Belgique: un préalable non rempli}

5 Pour la Belgique, nombreux sont les observateurs qui diagnostiquent et dénoncent l'absence d'une politique active de gestion des sols ${ }^{2}$. On explique parfois ce constat sur base des rapports que la population et ses représentants entretiennent avec la ressource foncière. Par exemple, lorsque les experts de l'ADEF $^{3}$ s'intéressent à la Belgique et commentent la faible intervention de la puissance publique sur les marchés fonciers, ils s'étonnent de l'idée prédominante selon laquelle le sol n'est pas, dans ce pays, considéré comme un bien rare et non reproductible, mais comme «un bien abondant que l'on peut consommer, ce qui peut engendrer une consommation excessive et un gaspillage de l'espace» (Acosta, 1994, p. 43). Pour un pays aussi densément peuplé, cette conception assez étonnante peut sans doute en partie s'expliquer par l'abondance des terres utiles et par la facilité technique de viabilisation avec laquelle la majeure partie du territoire peut recevoir des activités urbaines. Les Pays-Bas sont, à ce propos, fréquemment évoqués comme un exemple inverse où la lutte contre l'eau et la difficile réorganisation des systèmes de drainage font du sol un capital à consommer avec parcimonie (Vandermotten et al., 1999, p. 75). De nos travaux comparatifs, nous relevons que cette explication déterministe de la fragilité du milieu physique est également relevée par les auteurs ayant traité de l'endiguement urbain danois et suisse. En Suisse, en raison de l'étendue de la zone montagneuse, près de $80 \%$ des hommes et des activités se concentrent sur moins d'un tiers de la superficie du pays. Dans un tel contexte de compétition vive pour les basses terres et d'impossibilité pour l'agriculture de répondre à la demande, il n'est guère étonnant de constater que l'on y ait, très tôt, développé une tradition rigoureuse de planification (Werquin et Demangeon, 2000, p. 78). De même, au Danemark, la fragilité du milieu physique (7 300 kilomètres de littoral, faible altitude, présence de marécages, de dunes, de polders) et la volonté d'indépendance sur les plans alimentaire et énergétique ont, depuis longtemps, sensibilisé les populations à la nécessité d'une utilisation parcimonieuse de leur espace et de leurs ressources (Renard, 1995, pp. 1-3).

Préalablement à la loi organique de l'aménagement du territoire du 29 mars 1962, une relative anarchie régnait en Belgique dans le domaine de la planification spatiale (Vandermotten, 1982, p. 1). Avant la réalisation des 48 plans de secteur et la progressive introduction du zonage, la démarche d'aménagement consistait à autoriser les constructions à front de toutes les voiries pourvu qu'elles fussent équipées (Gosselain, 1999, p. 33). On retrouve dans cette démarche d'enrubanement si typique du territoire belge l'idée selon laquelle le sol est un bien abondant que l'on peut consommer. En effet, plutôt qu'une réflexion sur la nécessité de consommer une ressource rare afin de loger la population, on voit ici que ce sont les caractéristiques de l'offre qui définissent la légalisation des terres constructibles. Néanmoins, cette méthode était relativement économe en sol et en infrastructures aussi longtemps que la production de la ville était déterminée par des transports collectifs lents. Malheureusement, cela a commencé à poser problème lorsque la tradition de l'abondance foncière s'est maintenue 
parallèlement à la diffusion en masse de la conduite automobile, cela principalement en raison de la surabondance des zones d'habitat inscrites aux plans de secteur (Géron, 1997; De Decker, 2002). Lorsqu'il s'agit de rendre compte du gonflement exagéré des zones urbanisables et de la manière dont la réalisation des plans de secteur a permis à la périurbanisation de perdurer et de s'amplifier, la littérature épingle l'entremêlement de nombreux facteurs explicatifs. On peut tout d'abord lire que les plans de secteur sont la traduction spatiale héritée du modèle de développement keynésien des trente glorieuses et, dès lors, sont surdéterminés par les conditions du développement économique de l'époque (Schoonbrodt, 1975; Vandermotten, 1982; Géron, 1997). Face à cette logique de croissance et de "progrès», les limitations à caractère environnemental n'ont été que marginales et les objectifs écologiques envisagés davantage comme contraintes à respecter qu'en tant qu'objectifs fondamentaux (Vandermotten, 1982, p. 1). Parallèlement, sur ce thème des liens entre aménagement et environnement, il apparaît que les différentes contraintes du milieu physique - les zones inondables, la pente et la stabilité, la vulnérabilité des nappes aquifères - n'ont été que très partiellement prises en compte. Pour expliquer le surdi-mensionnement des zones urbanisables, on épingle également l'influence du lobby du génie civil (Vandermotten, 1982, p. 4), utilement relayée dans l'administration de l'Urbanisme par des fonctionnaires à la fois proches du Ministère des Travaux Publics et persuadés de la pertinence des thèses fonctionnalistes (Schoonbrodt, 1987, p. 147). Dans le chef de cette administration, on a également relevé la crainte «qu'une trop forte diminution de l'offre de terrains à bâtir ne fasse monter le prix de ces terrains, rendant plus difficile le logement des familles» (Schoonbrodt, 1975, p. 19).

7 Parce qu'il rend constructible de nombreux terrains en en améliorant l'accessibilité, le relâchement des freins à la mobilité est un puissant générateur de plus-value foncière. En effet, lorsqu'une parcelle initialement vouée à une activité morphologiquement rurale intéresse la demande urbaine, la possibilité de capter une rente d'urbanisation surgit. Pour le contexte belge, on observe que la dynamique insufflée par la possible captation de cette rente est un facteur déterminant de l'intensité périurbaine. En effet, afin d'expliquer le gonflement exagéré de nombreuses zones d'habitat, il faut, bien sûr, aussi prendre en compte les démarches entreprises par les propriétaires. Afin de bénéficier du «bon classement», ces derniers sont massivement intervenus auprès des auteurs de projets lors des phases d'élaboration des plans de secteur. Les pressions, qui ont pu aller jusqu'à la malversation (Haumont, 1990, p. 422), sont notamment passées par différentes réclamations individuelles ${ }^{4}$. Parallèlement, il apparaît que le monde politique a été réceptif à l'action des lobbies fonciers. En effet, les plans de secteur ont été qualifiés de très peu démocratiques (Schoonbrodt, 1975; Vandermotten, 1982, p. 4) et de nombreux élus seraient intervenus afin de privilégier les intérêts de certains particuliers. In fine, de la littérature portant sur l'élaboration des plans de secteur, nous relevons que le rapport de force entre la dynamique centrifuge de la rente d'urbanisation et les régulations collectives de la planification a plutôt été favorable à la première. Face à des fonctionnaires craintifs vis-à-vis d'une hausse des prix et face à des décideurs politiques peu conscientisés aux conséquences du gaspillage de l'espace, l'action de propriétaires désireux de capter la manne céleste - ou plutôt foncière - de la rente d'urbanisation semble globalement avoir été couronnée de succès.

8 Le mécanisme juridique de l'indemnisation des servitudes d'urbanisme est également un facteur important à prendre en compte si l'on cherche à expliquer la configuration des zones urbanisables inscrites dans les plans de secteur. C'est la loi organique du 29 
mars 1962 qui a intégré une formule d'indemnisation suite à une interdiction de bâtir ou de lotir résultant d'un plan mettant fin à l'usage auquel un bien est affecté ou normalement destiné (Gabriel et al., 2001, p. 23) ${ }^{5}$. L'intégration spécifique d'une procédure d'octroi d'indemnisation aux propriétaires dont les biens allaient se trouver grevés de nouvelles servitudes d'urbanisme par les conséquences de la loi organique illustre parfaitement l'idée prédominante en Belgique selon laquelle le foncier est une marchandise abondante et une source de plus-values à valoriser. En effet, plutôt que la nécessité de répondre à une demande légitime, c'est ici, de nouveau, la logique de l'offreur foncier qui est prise en compte pour définir le caractère constructible des terrains. Assimilant la notion de "destination normale» à la notion de «terrain à bâtir», la jurisprudence et la doctrine se sont imprégnées du point de vue de l'offreur foncier pour dégager les trois conditions objectives qui définissent le terrain à bâtir: être apte techniquement à la construction, être voisin d'autres habitations ou d'autres terrains à bâtir et être situé le long d'une voirie équipée. Relayant la conception selon laquelle le sol est abondant, c'est l'application de ce principe de l'indemnisation des servitudes d'urbanisme qui explique en grande partie l'importance des formes urbaines linéaires et, parallèlement, la surabondance foncière qui caractérise de nombreux bassins résidentiels. En effet, lors de l'élaboration des plans de secteur, les pouvoirs publics ont affecté en zone ædificandi de nombreux terrains pour lesquels ils pouvaient craindre de devoir payer une indemnité (ADEF, 1983, p. 96; Haumont, 1990, p. 478).

Pour le contexte belge, la gestion de l'articulation entre l'offre potentielle définie par les plans de secteur et l'offre effective réellement disponible sur le marché des terres constructibles atteste aussi de la faible conscientisation au gaspillage de l'espace et à la concomitante dispersion de l'urbanisation. Transformer l'offre potentielle en offre effective afin de procéder à l'édification d'un immeuble implique de remplir deux conditions. Il faudra tout d'abord que le terrain soit mis sur le marché. En raison du comportement patrimonial des propriétaires, cela est loin d'être automatique. Transformer l'offre potentielle en offre effective implique ensuite, et on le néglige trop souvent, un processus de production technique. Afin de limiter le gaspillage de la ressource foncière, un moyen efficace est que la collectivité assure elle-même la viabilisation technique de l'offre effective, cela par une politique de "production foncière» (Comby et Renard, 1996). C'est fréquemment de cette manière que les nouveaux développements urbains sont gérés dans les pays où l'on veille à ne pas gaspiller le foncier vierge, par exemple en Suède et aux Pays-Bas, via des réserves de terrain à long terme des villes en Suède et via un quasi-monopole de fait des municipalités sur la production des parcelles à bâtir aux Pays-Bas (Renard, 1999, p. 10). En Belgique, la tradition de "non-parcimonie " a induit d'autres modalités pour l'articulation entre l'offre potentielle et l'offre effective. Nous sommes ici en présence d'une politique «d'offre foncière» (Comby et Renard, 1996), ce qui implique une délimitation publique de l'offre juridiquement urbanisable, mais ensuite la nécessité d'une initiative privée lorsqu'il s'agit de transformer l'offre potentielle en offre effective. Le poids laissé à l'initiative privée dans le processus d'urbanisation montre que la puissance publique ne juge pas nécessaire de canaliser les nouveaux développements en s'assurant que les terrains potentiellement urbanisables soient effectivement mis en œuvre. En effet, de cette rétention spéculative pratiquée par de nombreux propriétaires, et cela notamment pour certains terrains situés au sein même ou à proximité immédiate des agglomérations, il en résulte immanquablement un gaspillage de l'espace, un étalement de l'urbanisation et un mitage du territoire. 


\section{L'annihilation de la dynamique centrifuge de la rente d'urbanisation en Europe du Nord}

10 Au Danemark, pays où l'on s'efforce de ne pas gaspiller le sol, l'adéquation entre l'offre potentielle et l'offre effective s'opère dans un contexte général de propriété privée des terrains, à la différence des Pays-Bas ou de la Suède voisine. Plutôt qu'une production foncière publique massive, c'est ici l'outil fiscal qui permet de favoriser la réalisation des zones potentiellement urbanisables, via notamment les deux outils de la taxe d'urbanisation (Frigørelsesafgift) et de la taxation sur la valeur de site (Grundskyld) (Renard, 1995). Le Grundskyld est un impôt foncier dont l'originalité réside dans la base d'imposition. En effet, l'assiette fiscale ne dépend pas des constructions et n'est déterminée que par la localisation et par les règles d'urbanisme afférentes. Naturellement, cette taxation des terrains en fonction du «meilleur usage possible» constitue une puissante incitation à la réduction des divers comportements spéculatifs. A propos du Grundskyld, on peut encore remarquer qu'il s'agit là d'un outil hérité de pratiques fiscales basées sur la productivité différentielle des terres agricoles, ce qui renvoie aux relations entre la fragilité du milieu physique et la rigueur de la gestion territoriale. Pour réduire la rétention sur l'offre potentiellement urbanisable, la taxe d'urbanisation (Frigørelsesafgift) est un élément essentiel du dispositif danois. Elle peut représenter $60 \%$ de la plus-value et le propriétaire doit s'en acquitter dès qu'un terrain passe au plan d'urbanisme du statut de terre agricole à celui de terrain urbain. Naturellement, cela le pousse à directement alimenter le marché des terres constructibles. Si les capacités financières du propriétaire ne lui permettent pas de s'acquitter de cette taxe, la commune est alors dans l'obligation d'acheter le bien, ce qui a pour effet de restreindre la revendication en zones urbanisables de la part des municipalités, qui ne désirent pas grever leur budget par des acquisitions foncières inutiles. En plus d'un système sophistiqué d'évaluation foncière, l'usage opérationnel de ce mécanisme fiscal oblige donc les autorités locales danoises à une connaissance de la demande foncière et à la prévision fine des besoins réels en nouveaux logements. Le mécanisme de la taxe d'urbanisation a également pour effet de limiter la pression des propriétaires envers l'autorité planificatrice. En effet, cumulée avec la participation aux coûts des équipements qui est due lors de l'obtention des permis de construire, cette taxe finement calculée réussit le tour de force de neutraliser l'effet du zonage sur les valeurs foncières (Renard, 1995, p. 40). En d'autres termes, cette taxe annihile la dynamique centrifuge de la rente foncière d'urbanisation.

11 Dans nos sociétés occidentales modernes, le droit de propriété correspond à un élément essentiel, par ailleurs considéré comme une légitime liberté individuelle devant être protégée par l'autorité publique. A ce propos, 1789 sert souvent de repère et la «Déclaration des droits de l'homme et du citoyen» accorde à la propriété foncière un statut de droit «inviolable et sacré» (ADEF, 1989). Pour autant, comme le précise l'article 544 du Code civil ${ }^{6}$, la possibilité de jouir de sa propriété n'est absolue que par rapport aux règlements mis en place pour la réguler. Le caractère plus ou moins poussé de ce droit évolue donc en fonction des lois qui, elles-mêmes, dépendent des besoins et des exigences de la société. Le contenu du droit de propriété renvoie en fait à différentes catégories. John Christman (cité par Krueckeberg, 1995, p. 38) en identifie neuf: la possession, l'usage, les droits d'aliéner, de consommer, de modifier, de détruire, de gérer, d'échanger et le droit aux plus-values. Par exemple, si la propriété d'un 
immeuble classé ou d'un terrain signifie possession et usage, cela n'autorisera pas pour autant le propriétaire à les modifier sans respecter les règlements afférents. En référence aux mécanismes fonciers et immobiliers, le droit de propriété peut se lire comme une composante individuelle. A cette dimension individuelle de jouir pleinement de sa propriété, s'opposent les deux dimensions collectives de la planification spatiale et de la collectivisation de la rente, ce qui explique pourquoi la puissance publique doit intervenir par une politique foncière et immobilière. L'arbitrage entre liberté individuelle et régulation collective est dès lors inévitable et le point d'équilibre sera en permanence renégocié, au fur et à mesure des évolutions technologiques, des changements sociaux et des rapports de force sociopolitiques (Jacobs, 1997, p. 58). De nos comparaisons entre le contexte belge et des pays comme le Danemark ou les Pays-Bas, nous relevons un différentiel majeur quant à la légitimité de laisser au propriétaire foncier le bénéfice de la rente lorsque le terrain passe d'un usage rural à un usage urbain. Cette conclusion s'inscrit dans les analyses développées par Renard (1999, p. 10) lorsqu'il observe qu'à ce propos deux conceptions de la propriété foncière coexistent en Europe. Dans les pays d'Europe du Nord, la conception largement répandue est que la propriété $d u$ sol n'inclut pas le droit à la plus-value résultant du mouvement général d'urbanisation. Dans le cas des Pays-Bas ou de la Suède, on ne s'enrichit pas dans le foncier puisque le surprofit est directement capté par la collectivité via la production foncière. Pour le Danemark, nous avons vu que c'est l'intégration des outils de la planification physique et d'une fiscalité incitative qui permet de neutraliser les soubresauts de la rente. Par contre, dans les pays d'Europe du Sud, on observe, en général, une conception très respectueuse du droit de propriété, qui consiste à laisser au propriétaire initial le bénéfice de la plus-value, sous réserves toutefois de corrections fiscales. De ce point de vue, un pays comme la Belgique appartient clairement au modèle «méridional». L'idée selon laquelle il est tout à fait légitime de faire fructifier son patrimoine foncier semble, en effet, clairement inscrite dans les mentalités.

\section{La limitation de la zone juridiquement constructible en Suisse}

En Suisse, il n'existe pas de réelle politique foncière active visant, à l'instar du Danemark, à restreindre la rétention spéculative des propriétaires ou, à l'instar des Pays-Bas et de la Suède, à rendre publique la viabilisation technique des sols. En Suisse, nous observons, comme en Belgique, un décalage entre les zones à bâtir légalisées et l'offre immédiatement disponible à la construction (Ruegg, 2000, p. 156). Dans ce pays, on observe également, comme en Belgique, une influence des propriétaires sur l'autorité en charge du zonage. A ce propos, nous lisons par exemple que de nombreuses communes ont conscience du risque que le zonage introduise une inégalité entre propriétaires et que, «pour le prévenir, il n'est pas rare de découvrir dans les plans d'affectation que chaque propriétaire foncier a été servi. L'autorité a veillé à ce que chacun - ou en tout cas le plus grand nombre - ait du terrain en zones à bâtir» (Ruegg, 2000, p. 147). Par rapport à la Belgique, on observe toutefois trois facteurs importants de limitation de la zone juridiquement constructible. La limitation tient tout d'abord à la volonté politique de préserver $4500 \mathrm{~km}^{2}$ de surfaces d'assolement agricole (SDA), soit $13 \%$ du territoire. Le plan sectoriel des surfaces d'assolement, qui a pour origine la rareté des «terres utiles», est un document de planification élaboré par la Confédération pour satisfaire à la légitimité abstraite d'assurer l'auto- 
approvisionnement du pays en cas de conflit. Sans l'intervention de l'Etat, une partie de cette superficie serait disponible pour la construction (Ruegg, 2000, p. 153). Il existe en Suisse un second facteur de limitation de la zone constructible déterminé par les caractéristiques du milieu physique, celui de la planification des infrastructures. En effet, la desserte de nombreux noyaux d'habitat y nécessite la mise en place de canalisations dans des terrains escarpés et rocheux et les coûts qui en résultent expliquent la volonté de limiter l'éparpillement (Sayagh, 1991, p. 49). En parallèle, la présence de nombreux lacs à l'aval des bassins versants explique pourquoi la mise en place des infrastructures nécessaires au traitement des eaux usées est une priorité déjà ancienne. C'est en effet en 1955 qu'a été mise en place la loi fédérale sur la protection des eaux. Cette législation a été un élément déterminant dans la lutte des aménageurs contre la dispersion de la maison individuelle (Garnier, 1984, p. 54) puisque les contraintes financières font que la production de l'urbanisation n'aboutit pas à la périurbanisation diffuse lorsque l'on tient à traiter les eaux usées au sein de stations d'épuration collectives (Halleux, 2001b). Un troisième facteur limitatif de l'offre potentielle est en Suisse celui de la non-indemnisation des servitudes d'urbanisme (Moor et Donzel, 1999). Sur ce plan, les situations belge et suisse sont donc opposées puisque les autorités planificatrices helvètes sont autorisées à «déclasser» des zones urbanisables sans, pour autant, devoir verser des indemnités pour moins-values. Dans les faits, lorsqu'il s'est avéré que les zones à bâtir décidées sous le régime antérieur étaient surdimen-sionnées, les communes ont ainsi pu, sans crainte pour leurs budgets, réduire les zones urbanisables pour les affecter à une autre destination. La logique sous-jacente à ce mécanisme juridique consiste à différencier la propriété du bienfonds au sens formel de la propriété de l'usage du sol (Ruegg, 2000, p. 144). En d'autres termes, même si la dynamique centrifuge de la rente foncière n'est pas aussi bien maitrisée que dans les parties plus septentrionales du continent, priver les propriétaires de la rente d'urbanisation en supprimant le droit d'urbaniser leurs terrains n'est pas, en Suisse, considéré comme une atteinte au droit de propriété. Contrairement à la Belgique, les exigences d'une régulation collective comme celle de l'aménagement prévalent sur cette composante du droit de propriété. In fine, de la comparaison entre la Belgique et la Suisse, nous relevons que le meilleur endiguement des villes suisses résulte, certes, des contraintes du milieu physique, mais également d'une conscientisation plus poussée aux nécessités de la planification et d'une prégnance moindre du droit de propriété. L'arbitrage entre droits individuels et droits collectifs aboutit à un autre point d'équilibre qu'en Belgique, un point d'équilibre plus en faveur du maintien de la compacité urbaine.

\section{Pourquoi cette tradition belge de la non-gestion foncière?}

13 Au-delà des caractéristiques de l'environnement naturel précédemment commenté, d'autres facteurs peuvent-ils expliquer cette caractéristique si profonde de la société belge selon laquelle il n'est pas immoral de considérer le terrain à bâtir comme une marchandise ordinaire et comme une source de plus-value à valoriser? Pour Ph. Doucet (1985, p. 71), cette situation tient aussi au facteur historique de la longue intégration entre les milieux d'affaires et la propriété foncière. Cette fusion, dont l'histoire reste à écrire, s'explique notamment par les scrupules religieux de la classe agraire qui, lors de la vente des biens nationaux, lui auraient empêché de s'approprier le gigantesque capital foncier alors mis en vente, cela au profit d'une pragmatique bourgeoisie 
d'affaire. Il en aurait ainsi résulté une énorme puissance pour la grande propriété foncière au moment où, vers le début du XIX ${ }^{e}$ siècle, le pays débute son essor industriel (Pirenne, 1975, p. 186). L'importance de ces événements sur l'urbanisation diffuse qui affecte le territoire belge tient tout d'abord au pouvoir d'influence politique de la grande propriété. Il suffit, pour s'en convaincre, de suivre avec Ph. Doucet (1985) l'histoire des obstacles au changement dressés sur le chemin des réformateurs fonciers. Sur un plan plus idéologique, c'est la politique menée dans le domaine du logement qui, dès la loi de 1889 sur les habitations ouvrières, a permis à la grande propriété d'exercer son ascendant sur la société belge. C'est en effet à partir de cette époque que l'accession à la propriété se développe comme le pilier principal de la politique du logement, cela notamment afin de favoriser l'adhésion des ouvriers les mieux éduqués aux idéaux de la classe dominante (Kesteloot et De Maesschalck, 2001, p. 44). Il en a précocement résulté, via divers outils d'aides à la pierre et à la personne, la constitution d'une classe de petits bourgeois bailleurs (Doucet, 1983) et d'une masse de propriétaires occupants qui, cette fois pour des raisons électorales, ont également limité la pugnacité politique des réformateurs fonciers. En fin de compte, une telle politique longue et continue d'aide à l'accession et de valorisation de la propriété a conduit à l'édification progressive d'un contexte culturel où le moyen réputé le plus respectable de gérer son patrimoine en «bon père de famille» est d'investir dans la brique, cette fameuse «brique dans le ventre» qui caractérise désormais la population belge.

\section{Les préférences résidentielles, la politique du logement et la production immobilière}

14 Après avoir précisé les raisons de l'absence d'une politique de gestion des sols, nous pouvons maintenant poursuivre notre réflexion sur les causes de l'intensité périurbaine belge par la prise en compte des préférences résidentielles. Si nous savons maintenant pourquoi les populations disposent de très vastes territoires, encore faut-il préciser les raisons qui les poussent à quitter aussi massivement les noyaux urbains afin d'accéder au modèle périurbain de la maison individuelle isolée et dotée d'attributs ruraux. Des comparaisons internationales, il ressort que les préférences centrifuges qu'expriment très massivement les populations belges s'expliquent tout d'abord par une aspiration ancienne à la maison individuelle et au coin de nature qui peut y être associé. Toutefois, au-delà de ce premier facteur, nous verrons qu'il existe également en Belgique une longue élaboration sociale de ce modèle d'habitat. Plus qu'ailleurs, la puissance publique y est, en effet, massivement intervenue afin de renforcer la périurbanisation. En outre, nous verrons également que les interventions de la puissance publique ont contribué à la mise en place d'une dynamique qui impose le produit immobilier de la maison unifamiliale située en milieu périurbain.

\section{Des réticences anciennes pour les modes de vie urbains}

Une première raison qui pousse les Belges à «profiter» des territoires potentiels ouverts par la mobilité relâchée et par la faible gestion foncière correspond à leur préférence profonde pour l'habitat unifamilial. Afin d'expliquer cette situation, il est utile de se pencher sur les comparaisons européennes présentées dans le tableau 1 où l'on observe que les pays se différencient nettement sur la part de ménages en maison 
individuelle. A cette échelle d'analyse, rendre compte de la constitution des parcs de logements a conduit différents analystes à souligner les rapports que les populations entretiennent avec la culture urbaine. Afin d'expliquer la domination des appartements dans les parcs espagnols et italiens, c'est ici le modèle méditerranéen de la tradition des fortes densités qu'il faut souligner. Comme P. Merlin (1998, pp. 85-87), nous relevons, en effet, que les sociétés méditerranéennes ont toujours accordé une place prépondérante à la ville, cela dans un entremêlement de facteurs naturels - le climat chaud ayant pu conduire à privilégier des formes resserrées et des rues étroites -, anthropologiques (Hall, 1966, p. 177) et historiques. Une culture urbaine fortement enracinée est également à relever pour expliquer le poids de l'habitat collectif en Suisse, en Allemagne et en Autriche. A ce propos, nous pouvons nous appuyer sur l'explication culturaliste du modèle «rhénan» développée par J. Lévy (1997, p. 54, p. 142), explication qui articule les modalités de la constitution des villes à la coupure entre les trames rurales de l'habitat groupé et de l'habitat dispersé. Dans les parties de l'Europe - Est de la France, Europe centrale - où les populations ont dû se regrouper en raison de la récurrence des risques d'invasions et de guerres, la présence de voisins proches aurait induit une habitude des règles contraignantes de l'agglomération et une discipline collective mieux adaptée à la concentration urbaine. Comme sur les bords de la Méditerranée, il en a résulté une culture urbaine forte. A la valorisation urbaine des modèles méditerranéen et rhénan, s'oppose l'identité particulière de l'Ouest européen, de la Scandinavie au Nord du Portugal, en passant par les Iles Britanniques et la Belgique. Ce «monde "bocager" de l'Europe du Nord-Ouest, mieux protégé des invasions et aux organisations productives plus familiales, est demeuré jusqu'à aujourd'hui plus réticent vis-à-vis des modes de vie plus urbains, qu'il perçoit comme une contrainte pénible. Le modèle du pavillon individuel, en propriété et entouré d'un jardin, rencontre dans ces régions, avec ses variantes suburbaines et périurbaines, un succès spectaculaire» (Lévy, 1997, p. 142). Les travaux réalisés par M. Wiel (1999, pp. 34-37) sur la France confirment l'importance du substrat culturel. En effet, la ventilation entre habitat collectif et habitat individuel des villes françaises reflète parfaitement la typologie européenne, dont la principale ligne de partage se déduirait de la proximité littorale prépondérante Atlantique - Mer du Nord versus Méditerranée. La France, partie la plus étroite de l'isthme européen, serait ainsi «à cheval» sur les trois types de configurations de l'Europe du Nord-Ouest (de Bordeaux à Valenciennes), de l'Europe méditerranéenne (triangle Toulouse-Lyon-Nice) et de l'Europe rhénane (triangle Reims-Mulhouse-Strasbourg). 
Tableau 1. Comparaison internationale des parcs de logement.

\begin{tabular}{|c|c|c|c|c|c|}
\hline & $\begin{array}{l}\text { Part de la } \\
\text { maison } \\
\text { individuelle }\end{array}$ & $\begin{array}{l}\text { Part de } \\
\text { propriétaires } \\
\text { occupants }\end{array}$ & $\begin{array}{l}\text { Locataire } \\
\text { du secteur } \\
\text { privé }\end{array}$ & $\begin{array}{l}\text { Locataire } \\
\text { du secteur } \\
\text { public }\end{array}$ & $\begin{array}{c}\text { Part de } \\
\text { logements } \\
\text { déficients }\end{array}$ \\
\hline Belgique & $79 \%$ & $67 \%$ & $10 \%$ & $23 \%$ & $8 \%$ \\
\hline Pays-Bas & $73 \%$ & $65 \%$ & $28 \%$ & $6 \%$ & $13 \%$ \\
\hline Danemark & $71 \%$ & $49 \%$ & $13 \%$ & $38 \%$ & $8 \%$ \\
\hline Portugal & $61 \%$ & $53 \%$ & $19 \%$ & $26 \%$ & $6 \%$ \\
\hline Norvège & $61 \%$ & $65 \%$ & $15 \%$ & $3 \%$ & $20 \%$ \\
\hline France & $58 \%$ & $59 \%$ & $19 \%$ & $3 \%$ & $5 \%$ \\
\hline Suède & $56 \%$ & $54 \%$ & $22 \%$ & $18 \%$ & $6 \%$ \\
\hline Autriche & $54 \%$ & $42 \%$ & $17 \%$ & $23 \%$ & $1 \%$ \\
\hline Allemagne & $48 \%$ & $50 \%$ & $29 \%$ & $10 \%$ & $6 \%$ \\
\hline Espagne & $46 \%$ & $39 \%$ & $37 \%$ & $24 \%$ & $5 \%$ \\
\hline Italie & $36 \%$ & $85 \%$ & $14 \%$ & $1 \%$ & $9 \%$ \\
\hline Suisse & $32 \%$ & $70 \%$ & $20 \%$ & $4 \%$ & $9 \%$ \\
\hline Grande-Bretagne & $21 \%$ & $31 \%$ & $67 \%$ & $2 \%$ & $1 \%$ \\
\hline
\end{tabular}

Référence : début de la décennie 1990

Source : P. de la Morvonnais, 1998

\section{Des politiques rémanentes en faveur de la dispersion}

En comparaison des contextes méditerranéen et rhénan - notamment en Suisse -, une préférence ancienne de la population pour l'habitat individuel apparaît comme une explication recevable du faible endiguement des agglomérations belges. On peut supposer que ce substrat anti-urbain explique pourquoi, lors de la seconde moitié du $\mathrm{XIX}^{\mathrm{e}}$ siècle, la bourgeoisie belge a été rapidement séduite par les premières sirènes périphériques et par le modèle anglo-saxon de la "gentry» rurale plutôt que par les grandes opérations urbanistiques d'appropriation des quartiers centraux. Pour autant, la réticence anthropologique vis-à-vis des modes de vie urbains qui caractériserait la Belgique ne peut, à elle seule, rendre compte du mode de peuplement diffus qui l'affecte. En comparaison d'autres territoires immergés dans le même contexte «atlantico-nordique» - notamment la Grande-Bretagne, les Pays-Bas ou le Danemark -, la dispersion et l'éparpillement de l'habitat individuel y sont, en effet, nettement plus poussés. Pour rendre compte de cette situation, il faut cette fois remonter aux choix politiques opérés lors de la seconde moitié du XIXe siècle (Dubois, 2001, p. 97 et 2002). En effet, c'est à cette époque que se mettent en place les politiques publiques ayant conduit à la promotion de la maison individuelle (Mougenot, 1988) et à l'idéologie aujourd'hui dominante qui fait de la campagne le lieu de résidence idéal (Brück et al., 2001a). Parmi les choix politiques spécifiques à la Belgique et ayant façonné les actuelles dynamiques centrifuges et renforcé la valorisation du monde rural, il faut notamment relever, dès 1870 , l'introduction des abonnements ouvriers (Juchtmans et al., 1999, p. 10), cela afin de limiter la concentration des populations pauvres dans les taudis urbains. En s'appuyant sur un réseau dense de chemins de fer et de tramways vicinaux, ces abonnements ont, dès la fin du XIX ${ }^{\mathrm{e}}$ siècle, permis de concilier, d'une part, la vie traditionnelle et bon marché de la campagne et, d'autre part, le travail fixe rémunéré au sein des villes et des centres industriels naissants. En comparaison des autres pays européens, les longs mouvements pendulaires et le concomitant ralentissement de la croissance des noyaux urbains sont donc, en Belgique, antérieurs 
de plusieurs décennies. A ce propos, l'analyse du cas danois indique qu'il faut attendre les années 1920-1930 pour que l'amélioration des techniques de transport ralentisse la croissance des zones centrales de Copenhague (Andersen, 1991, pp. 368-369). Identifier les raisons précises du renforcement du substrat anti-urbain belge nécessite de considérer les fondements sociopolitiques de cette mesure sur les abonnements ouvriers. A l'instar des facteurs explicatifs relevés ci-dessus sur la prégnance de la propriété foncière, on est ici, de nouveau, en présence de facteurs entremêlant l'action d'une partie de la bourgeoise et l'influence de l'Eglise. Nous constatons, en effet, qu'un objectif majeur de cette mesure fut de limiter une croissance urbaine susceptible d'alimenter le mouvement socialiste au dépens de l'influence catholique et des intérêts de la classe dominante (Kesteloot et De Maesschalck, 2001, p. 43).

L'articulation entre les choix politiques de la fin du XIX ${ }^{e}$ siècle et l'actuelle diffusion périurbaine implique également de prendre en compte les choix opérés en matière d'aide au logement ${ }^{7}$. En effet, depuis la loi du 9 août 1889 inaugurant la politique sociale du logement, l'action des pouvoirs publics belges a consisté en une aide rémanente à l'accession à la propriété et, corollairement, à la construction neuve hors ville. Afin d'expliquer l'orientation politique précoce en faveur du statut de propriétaire, nous pouvons nous remémorer le poids de la grande propriété commenté ci-dessus et rappeler la volonté de contrôle social manifestée par la bourgeoisie et par l'Eglise (Kesteloot et De Maesschalck, 2001, p. 43). Pour l'autorité civile et le pouvoir économique, multiplier le nombre de propriétaires apparaît, en effet, comme un moyen efficace d'orienter la classe ouvrière vers des préoccupations «sereines». Pour l'Eglise, il s'agit là de valoriser les valeurs domestiques et familiales aux dépens des valeurs collectives. Cette promotion de la propriété, qui ne sera jamais fondamentalement remise en cause par l'association des socialistes au pouvoir, s'est traduite par la mise en place de multiples outils bien connus sur lesquels il n'est pas utile de s'étendre (développement du marché du crédit hypothécaire, construction par des sociétés publiques, octroi de primes, prêts à taux réduits). Il est toutefois intéressant de relever le sommet que constitue, en 1935, la création d'un outil comme la Société Nationale de la Petite Propriété Terrienne. Créée à l'initiative d'une coalition catholique - libérale, il s'agit alors de proposer un mode de vie familial et rural via le financement de constructions de maisons individuelles destinées à la vente.

Face à la rémanence de l'aide à la propriété, l'aide à la location est historiquement peu présente en Belgique. Elle est quasi nulle quant au volet aide à la personne (Mougenot, 1987, p. 78) et, en comparaison internationale, l'offre locative publique est très réduite. Des données rassemblées dans le tableau 1, on constate notamment que, sur ce point, la situation belge diffère fortement de celle des deux voisins "atlantiques» que sont le Danemark et les Pays-Bas. Par rapport à ces pays, où l'effort de reconstruction qui suivit la Seconde Guerre Mondiale fut massivement marqué par l'intervention publique (Boelhouwer et van der Heijden, 1992), la Belgique a pu, lors de cette période, s'appuyer sur la tradition des initiatives privées. C'est d'ailleurs à cette époque que la conjonction de la tradition pour l'aide à la construction neuve hors ville et des dynamiques de la croissance économique keynésienne enclenche la périurba-nisation diffuse de masse ${ }^{8}$. Historiquement, le pilier «accession à la propriété» de la politique du logement n'est pas lié au seul volet de l'occupation. En effet, de nombreux outils mis en place depuis 1889 ont également permis à des petits bourgeois de placer leurs économies dans la construction neuve d'immeubles de rapport. Jusqu'aux années soixante, leur action 
participa également à la résorption relativement efficace des pénuries de logement, bien plus passagères en Belgique qu'ailleurs (Doucet, 1983). Toutefois, lors des dernières décennies, la maitrise d'ouvrage de la part des bailleurs s'est réduite, cela notamment en raison d'un différentiel de rentabilité de plus en plus favorable aux placements mobiliers. Concernant la politique du logement menée en Belgique, il faut aussi constater qu'elle est peu performante quant aux critères de l'entretien et de la rénovation du parc locatif existant (De Decker, 1990), ce dont atteste la part très importante de logements déficients ${ }^{9}$ (Tableau 1). En raison de l'étroitesse du parc public et de la raréfaction des investissements privés dans l'immobilier résidentiel locatif, cette situation se traduit aujourd'hui par de substantiels problèmes sociaux et par une accentuation de la polarisation sociale entre propriétaires et locataires (De Decker, 2002). Par rapport à la Belgique, l'intervention des autorités suisses et danoises dans le domaine de l'aide au logement s'inscrit dans un meilleur équilibre entre les deux secteurs de l'acquisitif et du locatif. Cet équilibre, qui tient à la fois aux politiques fiscales (aide moins importante aux accédants et moindre fiscalité immobilière) et aux outils explicites de la politique du logement ${ }^{10}$ a permis de maintenir la rentabilité de l'investissement immobilier locatif. Au Danemark comme en Suisse, il en résulte un parc locatif de meilleure qualité et les ménages ne sont pas, à l'instar des populations belges, contraints à l'accession pour disposer d'une offre adéquate.

\section{Les mécanismes de la production immobilière contemporaine}

19 Par rapport aux autres pays intégrés dans le contexte "Atlantique» de la maison unifamiliale, la faible intervention de la puissance publique dans la constitution d'un parc locatif n'est pas la seule spécificité belge. En matière de constitution des stocks de logements, il en existe une seconde qui correspond au rôle du premier occupant des immeubles dans leur production. Sur base de la figure 1, on constate qu'au sein des principaux pays occidentaux, c'est en effet à la Belgique que revient la palme de l'autopromotion $^{11}$. A la différence de la Belgique, la filière promoteur est par contre largement majoritaire au Royaume-Uni, aux Pays-Bas et au Danemark ${ }^{12}$. Rendre compte de l'importance de l'auto-promotion en Belgique oblige tout d'abord à revenir sur les marchés fonciers et sur la tradition d'abondance des terres à bâtir. En effet, une configuration foncière différente avec une offre plus rare et des prix plus élevés aurait sans doute induit d'autres modalités puisque l'on observe que ces deux facteurs poussent les professionnels à se lancer dans la maîtrise d'ouvrage. Pour les entreprises de construction, l'acquisition du terrain peut devenir une nécessité lorsque les particuliers ne sont plus aptes à lever l'obstacle foncier. Dans certaines régions de Belgique, désormais marquées par une pénurie d'offre effective, c'est cette situation qui explique pourquoi des entrepreneurs-promoteurs désireux d'alimenter leur activité développent des acquisitions foncières au service de leur propre maîtrise d'ouvrage. Une hausse des prix fonciers peut également pousser les professionnels à se lancer dans la maîtrise d'ouvrage, cette fois via des promoteurs-spéculateurs attirés par la possibilité de compléter la légitime marge de promotion par l'adjonction d'un important volume de rente. Diverses analyses nationales confirment cette articulation entre les mécanismes fonciers et les acteurs qui prennent l'initiative de la construction résidentielle. Que ce soit en France, au Royaume-Uni ou en Belgique, ce sont les zones excentrées aux marchés fonciers abondants et détendus qui connaissent la plus faible intervention des promoteurs dans la constitution du cadre bâti (Dubois, 2001, p. 43 et 
2002). En parallèle à la tradition de l'abondance foncière, la tradition de l'aide publique à l'accession pourrait également expliquer la spécificité belge de l'auto-promotion. En effet, nous avons vu ci-dessus que cette politique a conduit à la constitution d'une masse de petits propriétaires bailleurs dont l'action, à la sortie de la Seconde Guerre mondiale, a permis une résorption rapide de la pénurie. En plus d'avoir longtemps offert un substitut à un parc public très étroit, la maîtrise d'ouvrage de ces opérateurs aurait également retardé l'apparition du promoteur immobilier professionnel, contribuant par-là à limiter son degré d'influence dans la filière construction (Doucet, 1983, p. 10).

Figure 1. Part de l'auto-promotion durant la décennie quatre-vingt.

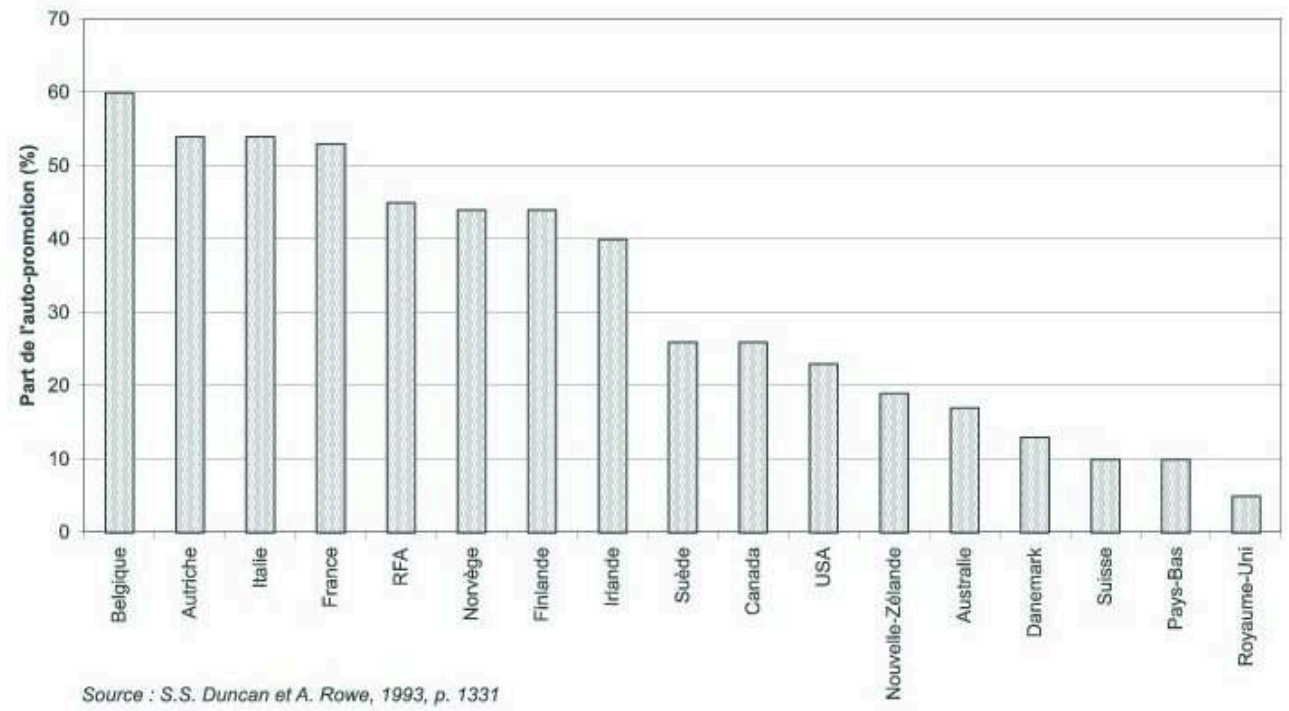

L'analyse réalisée par V. Kaufmann et al. (2001) sur le degré de liberté des choix résidentiels des périurbains français montre que nombre de ménages sont contraints par les mécanismes des marchés de l'immobilier. On retrouve en effet dans les périphéries françaises de nombreuses familles qui auraient souhaité rester dans un quartier central et qui ont été contraintes de s'exurbaniser faute d'une offre financièrement accessible correspondant à leurs désirs. De ce point de vue, la périurbanisation résidentielle contemporaine n'est pas que l'expression agrégée de désirs individuels, elle apparaît également comme le résultat d'une "dynamique totalisante à des répondants porteurs d'autres projets» (Kaufmann et al., 2001, p. 150). En raison de la structuration historique de la production immobilière, la situation est sans doute similaire en Belgique. En effet, l'étroitesse du parc locatif public, la dévalorisation du parc locatif privé et la faible influence des promoteurs dans la filière construction ne permettent pas non plus de répondre à l'aspiration des familles qui désireraient s'inscrire dans des modèles alternatifs à la maison unifamiliale périphérique. A l'inverse, en Suisse et au Danemark, on observe que les mécanismes de l'immobilier résidentiel ont permis la constitution d'une offre alternative. Dans ces pays, la possibilité de la villa périphérique existe, mais les migrations résidentielles d'agrandissement du ménage, qui constituent le vecteur traditionnel de la périurbanisation (Brück et al., 2001a), peuvent se diriger vers d'autres types de produits immobiliers. En Suisse, il s'agit essentiellement d'un parc locatif d'appartements de 
qualité. Au Danemark, il s'agit plutôt de maisons unifamiliales inscrites dans un tissu pavillonnaire dense et mises en place via la filière promoteur.

\section{Le choix clair de la Belgique en faveur de la mobilité voiture}

21 Expression brutale du relâchement des contraintes de la mobilité quotidienne, le phénomène périurbain s'intègre dans un contexte systémique où la propension à la conduite automobile joue un rôle premier. Face à un éclatement urbain particulièrement poussé, il est dès lors cohérent de retrouver en Belgique une très forte dominance de ce mode de transport, ce que confirment notamment les comparaisons internationales réalisées par l'OCDE ${ }^{13}$ ou les données sur les navettes de travail rassemblées dans le tableau 2. Rendre compte du haut degré de périurbanisation qui affecte la Belgique oblige dès lors à considérer les rapports que la population et ses représentants entretiennent avec la mobilité automobile.

Tableau 2. Les parts modales pour les migrations pendulaires de travail.

\begin{tabular}{lccccc} 
& A pied & $\begin{array}{l}\text { Vélo et } \\
\text { mobylette }\end{array}$ & $\begin{array}{l}\text { Véhicule } \\
\text { individuel } \\
\text { motorisé }\end{array}$ & Train & $\begin{array}{c}\text { Autres } \\
\text { transports } \\
\text { collectifs }\end{array}$ \\
\hline Belgique 1991 & $5,7 \%$ & $9,3 \%$ & $70,0 \%$ & $6,2 \%$ & $8,7 \%$ \\
Suisse 1990 & $10,1 \%$ & $10,4 \%$ & $51,6 \%$ & $11,2 \%$ & $16,7 \%$ \\
Danemark 1993 & $4,5 \%$ & $24,6 \%$ & $54,7 \%$ & $6,7 \%$ & $9,5 \%$
\end{tabular}

Source pour la Belgique: G. Juchtmans et al., 1999, p. 54

Source pour la Suisse: P.-A. Rumley, 1999, p. 246

Source pour le Danemark: Statistics Denmark

De manière générale, même si le tableau 2 atteste que la voiture est désormais le moyen de transport majoritaire dans les trois pays étudiés, il ressort également de ces données que son utilisation est significativement plus importante en Belgique. Au Danemark, l'utilisation modérée de la voiture est à mettre en parallèle avec un taux de motorisation relativement faible, de 340 voitures pour 1000 habitants, ce qui est à comparer aux valeurs suisses et belges qui sont respectivement de 462 et de $441^{14}$. La première explication à la faible motorisation des Danois correspond à une politique fiscale qui tend à décourager l'acquisition et, dans une moindre mesure, l'usage de la voiture particulière. Il s'agirait là de l'héritage d'une vision socio-démocrate qui considère la voiture comme un produit de luxe socialement indésirable, pensée qui perdure en dépit du très haut niveau de richesse. Par rapport à la moyenne européenne, le Danemark et la Belgique se trouvent ainsi dans des situations opposées quant au prix relatif de l'automobile. Les travaux comparatifs élaborés par A. Bieber et J.-P. Orfeuil (1993, p. 131) indiquent que pour une moyenne européenne de 100, le prix d'acquisition d'une voiture est de 131 au Danemark et de 84 en Belgique. Financièrement, l'utilisation des transports collectifs est par contre plus avantageuse au Danemark et la même comparaison intra-européenne affiche cette fois un niveau de 92 pour le pays scandinave et un niveau de 103 pour la Belgique ${ }^{15}$. L'utilisation limitée de la voiture individuelle au Danemark tient également à l'alternative que représente le vélo. A la différence de nombreux pays, où cet outil a été abandonné suite à la modernisation et à l'enrichissement de la société (Newman et Kenworthy, 1999, p. 206), 
le vélo a bénéficié d'une forte attention de la part des autorités publiques danoises, notamment en termes d'aménagements de voirie. Il en résulte aujourd'hui une limitation de l'usage automobile sur les courtes distances. Les mesures adoptées par les autorités danoises dans le domaine du transport des personnes sont sans conteste déterminantes dans le maintien de la compacité urbaine. En effet, il ressort de cette politique intégrée que de très nombreux ménages danois tendent à organiser leurs quotidiennetés en n'utilisant qu'une voiture, ce qui, en raison d'un taux d'emploi féminin très élevé ${ }^{16}$, oblige fréquemment à une localisation résidentielle proche des lieux d'emplois et des nœuds de transports collectifs.

23 A la différence du Danemark, la mobilité voiture n'est pas financièrement pénalisée en Suisse, comme l'indique le haut niveau de motorisation (462 voitures/1000 habitants). Pour autant, même s'ils possèdent une voiture, de nombreux Helvètes ne sont pas rebutés par l'utilisation des modes alternatifs, ce qui résulte notamment de l'efficacité des modes de transports collectifs au sein d'une structure urbaine articulée sur des quartiers denses où l'habitat collectif demeure largement majoritaire. Pour rendre compte de la faible utilisation de la voiture en Suisse, il faut également relever les importants efforts financiers que continuent d'assumer les pouvoirs publics pour maintenir et développer les transports urbains collectifs et le chemin de fer (Delacrétaz, 1998; Kaufmann, 2000). Cette volonté déjà ancienne de maintenir la densité des réseaux ferrés tranche avec la situation rencontrée au sein de nombreux pays européens, notamment la Belgique où les réseaux collectifs ont été largement démantelés pour être progressivement remplacés par le réseau routier le plus dense des pays de l'OCDE (OCDE, 1998, p. 74).

Ces quelques éléments de comparaison entre la Belgique, le Danemark et la Suisse s'inscrivent parfaitement dans les conclusions d'études ayant précédemment mis en évidence que l'intense utilisation de la voiture en Belgique est le résultat de décisions politiques ayant très largement favorisé ce mode de transports: «en Belgique, un choix clair est fait en faveur de l'offre routière» (Bieber et Orfeuil, 1993, p. 130). Afin d'expliquer ce choix politique relativement net, on peut tout d'abord rappeler les relations systémiques entre les formes urbaines et les demandes de mobilité. En effet, une décentralisation urbaine débutée dès la fin du XIX ${ }^{e}$ siècle a, sans conteste, alimenté de manière précoce une forte demande de mobilité. En outre, on observe également à partir des comparaisons avec le Danemark et la Suisse que les rapports de force sociopolitiques sont en Belgique bien plus favorables à l'influence du lobby automobile. A la différence notamment du Danemark, la présence de nombreuses unités de montage pourrait expliquer la puissance d'influence des constructeurs, comme cela a par exemple pu être établi explicitement pour les Etats-Unis (Goldsmith et Jacobs, 1982). De la comparaison avec la Suisse, nous relevons qu'il n'existe pas en Belgique une aussi longue prise en compte politique des effets environnementaux du trafic automobile. Dans les grandes agglomérations alémaniques suisses, c'est en effet dès les années septante que, sous la pression de référendums ou d'initiatives populaires, les municipalités se sont lancées dans des politiques visant à réduire le trafic automobile en milieu urbain et à améliorer l'offre de transports publics. On trouve à l'origine de ces politiques une action très efficace d'associations militant pour promouvoir une politique des transports basées sur le respect de l'environnement (Kaufmann, 2000, pp. 74-75). 


\section{Conclusion}

De la comparaison internationale présentée dans cet article, nous relevons que l'intensité périurbaine qui affecte le territoire belge nécessite de simultanément considérer l'enracinement des dynamiques centrifuges et la faiblesse des mécanismes de régulations collectives. En comparaison de la Suisse et du Danemark, on se doit tout d'abord d'évoquer la détermination du milieu naturel. Comme aux Pays-Bas, il existe dans ces deux pays des raisons physiques objectives qui expliquent que la planification y soit significativement mieux ancrée. Parmi les raisons profondes de l'intense périurbanisation belge, on trouve également une aspiration ancienne pour la maison individuelle. Inscrite dans le modèle individualiste de l'Europe Atlantique, la Belgique se différencie sur ce point des cultures rhénane et méditerranéenne, caractérisées par une plus forte disposition pour la vie urbaine. Si elle est «atlantique» par son orientation pour l'habitat unifamilial, la population belge est par contre «méridionale» quant à la conception qu'elle entretient avec la ressource foncière. Rendue possible par l'abondance des terres utiles, mais héritée de facteurs historiques que nous ne faisons encore qu'entrevoir, cette conception consiste à considérer le sol comme une marchandise ordinaire, comme une source de plus-value à valoriser. C'est en grande partie cette conception qui explique le succès mitigé de la tentative d'endiguement qu'a représenté l'élaboration des plans de secteur, cela en raison des revendications individuelles des propriétaires et de l'interventionnisme inconscient de certains politiciens, mais également en raison du risque juridique potentiel de l'indemnisation des servitudes d'urbanisme, cette institutionnalisation de la privatisation de la rente foncière d'urbanisation. L'histoire politique du troisième tiers du XIX ${ }^{e}$ siècle est également essentielle pour comprendre les racines de l'éclatement périurbain actuel. A ce propos, nous avons rappelé comment les influences sociopolitiques d'une partie de la bourgeoisie et de l'Eglise catholique ont conduit à cimenter le substrat anti-urbain, à renforcer les valeurs familiales et à établir une tradition d'aide à la propriété et à la construction neuve. A la sortie de la Seconde Guerre Mondiale, lorsque la consommation de masse se développa et lorsque la conduite automobile se banalisa, ces traditions bien établies ont, sans conteste, grandement déterminé les comportements individuels.

Par rapport à la Belgique, les dynamiques centrifuges du développement économique keynésien, de l'individualisme de masse et de la mobilité motorisée semblent avoir été territorialement mieux régulées en Suisse et au Danemark. En raison des relations systémiques entre mobilité et potentiel urbain d'accessibilité, ce sont notamment les actions publiques visant à favoriser les modes de transports alternatifs à la voiture qui permettent d'y limiter la périurbanisation anarchique. En effet, par rapport à la Belgique, les choix politiques des dernières décennies n'y ont pas aussi clairement favorisé la motorisation individuelle. Dans ces pays, la conscientisation plus précoce aux équilibres environnementaux, à l'économie des infrastructures et à la préservation de la ressource foncière ont également constitué de puissants freins à la centrifugeuse périurbaine. Les régulations collectives adoptées en matière de logement expliquent aussi pourquoi la Suisse et le Danemark sont moins affectés par la périurbanisation diffuse. La ressource foncière y étant mieux préservée et l'aide au logement ne se limitant pas uniquement à favoriser l'accession, il en a résulté la production d'un parc immobilier qui permet de répondre à l'aspiration des familles désirant s'inscrire dans 
des modèles alternatifs à la maison unifamiliale périphérique, ce qui apparaît par contre plus malaisé en Belgique.

Trouver des formes de développements urbains durables en contrôlant mieux la périurbanisation appelle à des arbitrages difficiles. S'il convient, certes, de répondre aux attentes individuelles, il faut également limiter les effets secondaires les plus dommageables d'une urbanisation non canalisée. Sur ce plan, inverser les puissantes dynamiques centrifuges à l'œuvre en Belgique ne sera pas chose aisée. Néanmoins, face à un éclatement urbain particulièrement poussé, on observe que des acteurs de plus en plus nombreux s'interrogent sur le type de ville et de société vers lesquelles conduisent la périurbanisation diffuse à la belge (Halleux et De Keersmaecker, 2002). Les décideurs publics étant de plus en plus conscients des atteintes à la durabilité qu'engendre cette production de l'espace, on peut penser que de nouvelles régulations collectives s'imposeront progressivement, régulations qui permettront d'inscrire l'évolution des villes dans les principes du développement durable si elles nous conduisent à retrouver le sens de la vie citadine et de l'urbanité17 ${ }^{17}$ cette forme de politesse - du grec polis - qui permet aux hommes de vivre ensemble.

\section{BIBLIOGRAPHIE}

ACOSTA R. (1994), Politiques foncières comparées: Belgique, ADEF, Paris.

ADEF (Association des Etudes Foncières) (1983), Les enjeux de la fiscalité foncière, ADEF, Paris.

ADEF (Association des Etudes Foncières) (1989), Un droit inviolable et sacré: la propriété, ADEF, Paris.

ANDERSEN H.P. (1991), «The political urbanization fringe development in Copenhagen», Espace, Populations, Sociétés, 2, pp. 367-379.

ANTROP M. (2000), «Geography and landscape science», Belgeo, 1-2-3-4, pp. 9-35.

BARTHE-BATSALLE H., BRÜCK L., DE KEERSMAECKER M.-L., GEORGES X., HALLEUX J.-M., LAMBOTTE J.-M., MARECHAL L. et ROUSSEAUX V. (2002), Les coûts de la désurbanisation, Etudes et Documents - CPDT 1, Ministère de Région wallonne, DGATLP, Namur.

BIEBER A. et ORFEUIL J.-P. (1993), «La mobilité urbaine et sa régulation: quelques comparaisons internationales», Les Annales de la recherche urbaine, 59-60, pp. 126-139.

BOELHOUWER P. et VAN DER HEIJDEN H. (1992), Housing Systems in Europe: Part I. A comparative study of housing policy, Housing and urban policy studies, Delft University Press.

BRÜCK L., MAIRY N., HALLEUX J.-M. et SAVENBERG S., sous la direction de MERENNESCHOUMAKER B. et VAN HECKE E. (2001a), Rapport final avec propositions, Projet «Les comportements résidentiels des ménages face à la problématique du développement durable», SSTC - Leviers d'une politique de développement durable, Université de Liège, SEGEFA - ISEG, Université de Liège KULeuven, inédit. Consultable sur Inter-net: http://www.ulg.ac.be/geoeco/segefa/

BRÜCK L., HALLEUX J.-M., MAIRY N. et MERENNE-SCHOUMAKER B. (2001b), L'intervention de la puissance publique dans le contrôle de l'étalement urbain. Deuxième partie: confrontation de la situation belge aux expériences suisses et danoises, Projet «Les comportements résidentiels des ménages face à la 
problématique du développement durable», SSTC - Leviers d'une politique de développement durable, Université de Liège, SEGEFA, inédit. Consultable sur Internet : http://www.ulg.ac.be/geoeco/ segefa/

BURNIAT A. et GRIGNARD B. (1994), Séductions et déconvenues de la vie en lotissement, Institut de Sciences sociales appliquées, Faculté d'Economie, de Gestion et de Sciences sociales, Université de Liège, inédit.

COLARD A. et VANDERMOTTEN Ch. (1996), «La périurbanisation de l'emploi dans les zones métropolitaines belges», Revue Belge de Géographie, 1-2-3, pp. 33-40.

COMBY J. et RENARD V. (1996), Les politiques foncières, Coll. «Que sais-je», PUF, Paris.

DANISH ENERGY AGENCY (1998), Combined Heat and Power in Denmark, Copenhagen.

DE DECKER P. (1990), «Housing and housing policy in Flanders (Belgium); confusion on the eve of a new decade», The Netherlands Journal of Urban and Regional Research, 5, 1, pp. 3-27.

DE DECKER P. (2002), «Le piège du logement se referme!», Les échos du logement, 3, pp. 1-20.

DELACRÉTAZ Y. (1998), Mobilité urbaine et politiques de déplacements - le cas de trois agglomérations suisses, thèse $\mathrm{n}^{\circ} 1788$, EPFL, Lausanne.

DE LA MORVONNAIS P. (1998), «Comparaisons internationales», Logement et habitat l'état des savoirs (sous la direction de SEGAUD M., BONVALET C. et BRUN J.), La Découverte, Paris, pp. 147-157.

DE LANNOY W. et DE CORTE S. (2000), «Urban studies of Belgian geographers at the turn of the millennium», Belgeo, 1-2-3-4, pp. 189-20.

DE LANNOY W. et KESTELOOT Ch. (1985), «Différenciation résidentielle et processus de ségrégation", La cité belge d'aujourd'hui: quel devenir?, Bulletin trimestriel du Crédit Communal de Belgique, $\mathrm{n}^{\circ} 154$, pp. 137-150.

DOUCET Ph. (1983), «Le déclin de la petite propriété bailleresse», Courrier hebdomadaire du CRISP, $n^{\circ} 995$.

DOUCET Ph. (1985), «La politique foncière, une nécessité oubliée?», Les Cahiers de l'Urbanisme, 6, pp. 65-78.

DUBOIS O. (2001), La construction résidentielle en Wallonie: analyse spatiale multiscalaire et logiques socio-économiques de localisation, Thèse présentée en vue de l'obtention du grade de docteur en environnement, IGEAT, ULB, inédit.

DUBOIS O. (2002), «Stratégies des acteurs de la construction résidentielle neuve et caractéristiques des espaces bâtis en Belgique», Belgeo, 4, pp. 318-331.

DUBOIS O., GABRIEL I., HALLEUX J.-M. ET MICHEL Q. (2002), Révision des plans de secteur et mécanismes fonciers en Wallonie: objectifs politiques, outils juridiques et mise en œuvre, Etudes et Documents - CPDT 2, Ministère de Région wallonne, DGATLP, Namur.

DUBOIS-TAINE G. (2002), Outskirts of European cities. State of the art report, Cost Action C10, European Commission, Brussels.

DUBOIS-TAINE G. et CHALAS Y. (sous la direction de) (1997), La ville émergente, Monde en cours, l'Aube.

DUNCAN S.S. et ROWE A. (1993), «Self-provided Housing: The first World's Hidden Housing Arm», Urban Studies, 30, 8, pp. 1331-1354. 
FAVARGER P. (1991), «Suisse - Le rôle des politiques dans le retournement des marchés», Etudes foncières, 53, pp. 45-48.

FAVARGER P., FERRO-LUZZI G., MAC FARLANE A., SCHMID G. et THALMANN P. (1997), L'accès à la propriété du logement, IREC - EPFL, Lausanne.

FAVARGER P. et MAC FARLANE A. (1996), «Aménagement du territoire et construc-tion: l'outil fiscal», Raisons et déraisons de la ville - Approches du champ urbain, Presses polytechniques et universitaires romandes, Lausanne, pp. 325-342.

GABRIEL I., HALLEUX J.-M., HERBIET M., MICHEL Q. et MERENNE-SCHOUMAKER B. (2001), Taxation et indemnisation des plus- et moins-values liées aux modifications du plan de secteur, Note au Gouvernement wallon, LEPUR, Université de Liège, inédit.

GARNIER A. (1984), Les nouvelles cités dortoirs, Presses Polytechniques Roman-des, Lausanne. GERON G. (1997), «Politiques développées par la Région wallonne en matière de rénovation urbaine et de redensification des centres anciens», De villes en villes, Les Dossiers Espace-Vie, $\mathrm{n}^{\circ} 6$, pp. 83-92.

GOLDSMITH W.W. et JACOBS H.M. (1982), «The Improbability of Urban Policy», Journal of the American Planning Association, pp. 53-66.

GOSSELAIN P. (1999), «Le plan de secteur: essai de bilan pour un nouveau départ», Les Cahiers de l'Urbanisme, 27, pp. 21-36.

HALL E.T. (1966), The Hidden Dimension, Doubleday \& $C^{\circ}$, New York (trad. en français La dimension cachée, 1971, Editions du Seuil, Paris).

HALLEUX J.-M. (2000), «Développement durable et organisation urbaine: le mot d'ordre de la ville compacte», Les Cahiers de l'urbanisme, 30, pp. 18-23.

HALLEUX J.-M. (2001a), «Evolutions des organisations urbaines et mobilités quoti-diennes: espace de référence et analyse des processus», L'Espace géographique, 30, 1, pp 67-80.

HALLEUX J.-M. (2001b), «Les surcoûts de l'étalement urbain en Wallonie», Etudes foncières, 94, pp. 18-21.

HALLEUX J.-M. et DE KEERSMAECKER M.-L. (2002), «Les coûts de la désurba-nisation: pour qui, comment, pourquoi?», in BARTHE-BATSALLE H., BRÜCK L., DE KEERSMAECKER M.-L., GEORGES X., HALLEUX J.-M., LAMBOTTE J.-M., MARÉ-CHAL L. et ROUSSEAUX V., Les coûts de la désurbanisation, Etudes et Documents - CPDT 1, Ministère de Région wallonne, DGATLP, Namur, pp. $123-128$

HAUMONT F. (1990), Les instruments juridiques de la politique foncière, E. Story-Scientia, Bruxelles.

IGEAT (1994), Bilan de l'ajustement des réalités urbanistiques aux options fondamentales et aux prescriptions réglementaires des plans de secteur du Brabant wallon, Ministère de la Région wallonne, Direction Générale de l'Aménagement du Territoire et du Logement, ULB, inédit.

JACOBS H.J. (traduit de l'anglais par RENARD V.) (1997), «Le culte de la propriété aux Etats-Unis», Etudes foncières, 77, pp. 55-58.

JUCHTMANS G., VAN DER HAEGEN H. et VAN HECKE E. (1999), Les migrations de travail, Monographie $n^{\circ} 11 \mathrm{~B}$ «Migrations alternantes» du Recensement Général de la Population et des Logements au $1^{\text {er }}$ mars 1991 (sous la direction de MERENNE-SCHOUMAKER B., VAN DER HAEGEN H. et VAN HECKE E.), INS, SSTC, Bruxelles, pp. 9-203. 
KAUFMANN V. (2000), Mobilité quotidienne et dynamiques urbaines. La question du report modal, Presses polytechniques et universitaires romandes, Lausanne.

KAUFMANN V., JEMELIN C. et GUIDEZ J.-M. (2001), Automobiles et modes de vie urbains: quel degré de liberté, La Documentation française, Paris.

KESTELOOT Ch. ET DE MAESSCHALCK S. (2001), «Anti-urbanisme in Flanders: the political and social consequences of a spatial class struggle strategy», Belgeo, 1-2, pp. 41-62.

KRUECKEBERG D.A. (1995), «La propriété foncière, un concept difficile», Etudes foncières, 69, pp. $34-41$.

LEVY J. (1997), L'Europe. Une géographie, Hachette, Paris.

MERENNE-SCHOUMACKER B. (1998), «L'éclatement des polarités commer-ciales: nouvel enjeu de l'aménagement du territoire», Bulletin de la Société géographique de Liège, 34, pp. 55-75.

MERLIN P. (1998), Les banlieues des villes françaises, La documentation française. Notes et études documentaires, $\mathrm{n}^{\circ}$ 5073-74, Paris.

MINISTRY OF HOUSING AND URBAN AFFAIRS (1999), Housing, building and urban affairs in Denmark, Ministry of Housing and Urban Affairs, Copenhagen.

MOOR P. et DONZEL V. (1999), «Suisse: l'indemnisation des servitudes d'urba-nisme», Droit et Ville, 48, pp. 283-315.

MOUGENOT C. (1987), «Logement, assistance et promotion en Belgique», Les Annales de la recherche urbaine, 33, pp. 78-86.

MOUGENOT C. (1988), «Promoting the single-family house in Belgium. The social construction of model housing», International Journal of Urban and Regional Research, 12, pp. 531-549.

NEWMAN P. et KENWORTHY J. (1999), Sustainability and cities, Island Press, Washington.

OCDE (1995), Transports urbains et développement durable, OCDE, Paris.

OCDE (1998), Examens des performances environnementales. Belgique, OCDE, Paris.

OFFICE FÉDÉRAL DU LOGEMENT (OFL) (1996), «L'habitat en Suisse», Bulletin du logement, 63, OFL, Granges.

PIRENNE H. (1975), Histoire de Belgique, La Renaissance du Livre, Bruxelles, réédition de 1975, Tome IV.

RENARD V. (1995), Politique foncière au Danemark. Planification, évaluation et fiscalité foncière, Paris, ADEF.

RENARD V. (1999), «Où en est le système des transferts de COS?», Etudes foncières, 82, pp. 8-16.

RUEGG J. (2000), Zonage et propriété foncière, ADEF, Paris.

RUMLEY P.-A. (1999), Planification territoriale - Cours d'aménagement du territoire destinés aux étudiants Architectes, Ingénieurs en génie civil et Ingénieurs en génie rural, Lausanne.

SAVENBERG S. et VAN HECKE E. (2001), De residentiële keuze binnen het kader van suburbanisatie. Instrumentarium om te verhelpen aan de voornaamste knelpunten (België - Nederland - Engeland), DWTC - Hefbomen voor een beleid gericht op duurzame ontwikkeling, Instituut voor Sociale en Economische Geografie, K.U.Leuven, inédit.

SAVENBERG S., VAN HECKE E., BRÜCK L., HALLEUX J.-M., MAIRY N. et MERENNE-SCHOUMAKER B. (2001), L'intervention de la puissance publique dans le contrôle de l'étalement urbain. Première partie: état de la question en Belgique, Projet «Les comportements résidentiels des ménages face à la problématique 
du développement durable», SSTC - Leviers d'une politique de développement durable, KULeuven Université de Liège, ISEG - SEGEFA, inédit. Consultable sur Internet: http://www.ulg.ac.be/ geoeco/segefa/

SAYAGH N. (1991), «Vingt-quatre mesures pour économiser le sol», Etudes foncières, 53, p. 49.

SCHOONBRODT R. (1975), Plans de secteur - aménagement du territoire - urbanisme, Fondation Travail-Université, troisième édition, Bruxelles.

SCHOONBRODT R. (1987), Essai sur la destruction des villes et des campagnes, P. Mardaga, Liège.

SPORCK J.A. et VAN DER HAEGEN H. (1985), "Quelle urbanisation pour la Belgique et ses régions?», Bulletin trimestriel du Crédit Communal de Belgique, 154, La cité belge d'aujourd'hui: quel devenir?, pp. 263-272.

VANDERMOTTEN Ch. (1982), «Pratique de l'aménagement du territoire et développement régional. Bilan critique pour une alternative», Notes de recherches de la Société Géographique de Liège, 2, pp. 1-15.

VANDERMOTTEN Ch., VERMOESEN F., DE LANNOY W. et DE CORTE S. (1999), «Villes d'Europe: cartographie comparée», Bulletin du Crédit Communal, 207-208.

WERQUIN A.-C. et DEMANGEON A. (2000), Comparaison des politiques publiques nationales, régionales ou locales cherchant à freiner l'étalement urbain, dans quatre pays européens - rapport 2 Grande-Bretagne, Danemark, Suisse, Atelier d'environnement Thalès et Ministère de l'Equipement, des Transports et du Logement, Direction Générale de l'Urbanisme, de l'Habitat et de la Construction. Plan Urbanisme Construction Architecture, Paris.

WIEL M. (1999), La transition urbaine ou le passage de la ville pédestre à la ville motorisée, $\mathrm{P}$. Mardaga, Sprimont.

\section{NOTES}

1. Voir par exemple la revue bibliographique internationale présentée dans: J.-M. Halleux, 2000. Pour la situation wallonne, voir notamment: H. Barte-Batsalle et al., 2002.

2. Voir notamment: Ph. Doucet, 1985; O. Dubois et al., 2002; P. De Decker, 2002.

3. L'Association des Etudes Foncières (ADEF) est une association française active dans la domaine de la recherche sur le foncier (http://www.foncier.org).

4. Par exemple, «lors de l'enquête publique, le nombre de réclamations individuelles pour l'ensemble du Brabant wallon s'élevait à 2710 pour 268655 habitants (31/12/1976): 1\% de la population a réclamé» (IGEAT, 1994, p. 189).

5. Le principe de l'indemnisation des servitudes d'urbanisme est désormais repris au sein des trois législations régionales.

6. Article 544 du Code civil: «La propriété est le droit de jouir des choses de la manière la plus absolue, pourvu qu'on n'en fasse pas un usage prohibé par les lois et les règlements».

7. Voir par exemple: W. De Lannoy et Ch. Kesteloot, 1985; C. Mougenot, 1987; P. Boelhouwer et H. van der Heijden, 1992; O. Dubois, 2001, 2002; Ch. Kesteloot et F. De Maesschalck, 2001.

8. A la sortie de la Seconde Guerre Mondiale, cette tradition s'affirme notamment grâce au renforcement des primes à la construction et au cautionnement par l'Etat des crédits hypothécaire («loi De Taeye» de 1948).

9. Est considéré comme déficient un logement manquant d'un ou de plusieurs éléments de confort. 
10. En Suisse, selon la nature de l'occupant, ce sont les mêmes aides qui facilitent l'accession à la propriété ou qui permettent de limiter les efforts locatifs, tel par exemple l'abaissement de base, une aide qui prend la forme d'avances remboursables dans le but d'alléger l'investissement, cela pour le futur propriétaire occupant comme pour le futur propriétaire bailleur. En plus de faciliter l'accession à la propriété, cette mesure permet donc de diminuer la pression sur le marché locatif en assurant la rentabilité de l'investissement avec des niveaux de loyers plus faibles. L'abaissement supplémentaire correspond à une seconde mesure qui concerne à la fois l'acquisitif et le locatif. Il s'agit ici d'une aide à fonds perdus prenant à la fois la forme d'une prime à l'acquisition et d'une aide locative à la personne. A l'instar du système suisse, le système danois dispose également d'un important volet dédié à la location, notamment une aide locative pour les plus faibles revenus et, parallèlement, une protection des propriétaires bailleurs afin d'assurer un rendement minimum du capital investi (Ministry of Housing and Urban Affairs, 1999, pp. 34-36). Au Danemark, l'équilibre entre les deux secteurs de l'acquisition et de la location tient également à l'importance du parc social (Tableau 1), parc social dont le niveau de confort n'est pas significativement inférieur aux logements occupés par leurs propriétaires. En Suisse, c'est un système de subventionnement qui a été mis en place pour pallier les difficultés des moins favorisés. Il consiste à accorder une aide financière aux propriétaires de logements mis en location aux populations à revenus modestes. Notons que ces logements «sociaux» possèdent la même qualité que le reste du parc.

11. Dans la filière de l'auto-promotion, c'est le premier occupant de l'immeuble qui assure la maîtrise d'ouvrage. Par contre, dans la filière promoteur, la maîtrise d'ouvrage n'est pas assurée par un particulier en vue de l'occupation, mais par un promoteur en vue de la commercialisation.

12. Au Danemark, même si l'auto-promotion ne représente que $13 \%$ de la production immobilière entre 1980 et 1989, il apparaît toutefois que cette filière est dominante pour les maisons isolées (Source: S.S. Duncan et A. Rowe, 1993, p. 1335).

13. Les travaux de l'OCDE relèvent qu'en Belgique, «les chiffres du trafic par habitant et par unité de PIB sont parmi les plus élevées de l'OCDE» (OCDE, 1998, p. 74).

14. Source: Eurostat 1999.

15. Ces comparaisons sont basées sur une étude de consommation réalisée par l'Office Statistique de l'Union Européenne. Cette enquête identifie, pour chaque pays, le niveau relatif de certains biens et services par rapport au niveau général des prix du pays considéré.

16. En 1999, le taux d'emploi féminin est de 71,6\% au Danemark et de 50,2\% en Belgique (Source: Eurostat).

17. «Le respect d'autrui et de soi-même qui s'appelle d'ailleurs, à juste titre, l'urbanité» (Giraudoux).

\section{RÉSUMÉS}

Par rapport à ses voisins européens, la Belgique apparaît marquée par une périurbanisation à la fois plus importante et plus éclatée. De cette situation résultent sans conteste de nombreux accrochages par rapport aux principes stratégiques du développement durable. Le présent article se propose d'étudier les causes de l'éclatement urbain qui affecte le territoire belge à la lumière d'une comparaison avec la Suisse et le Danemark, deux pays réputés pour avoir mis sur pied des politiques relativement efficaces de lutte contre l'étalement urbain. De cette comparaison appréhendée via les trois thématiques de la politique foncière, de la production immobilière 
résidentielle et de la mobilité voiture, c'est l'enracinement profond de la périurbanisation belge qui apparaît. L'éclatement urbain qui y affecte le territoire s'explique, en effet, par une grappe de facteurs où s'entremêlent l'influence du milieu naturel, des réticences anciennes pour les modes de vie urbains, une faible conscientisation aux enjeux de la planification et des choix politiques rémanents ayant fortement contribué au renforcement des dynamiques centrifuges.

In comparison with neighbouring countries, the peri-urbanisation process in Belgium is very important in many aspects. In relation with sustainable development strategic objectives, this is a major issue and numerous researches have shown that peri-urbanisation contributes to various collective costs. By comparing Belgium with the situation in Denmark and in Switzerland, two countries renowned for their relatively effective urban containment, the aim of this paper is to analyse why sprawl so deeply affects the Belgian territory. Three issues are taken into account for this comparison: land policy, housing development and the Belgian choice for car mobility. This triple analysis shows that peri-urbanisation processes are deeply implanted in Belgium. They are indeed related with the influence of natural environment, with old reticence for urban life, with a weak consciousness towards planning and with persistent political choices in favour of centrifugal dynamics.

\section{INDEX}

Keywords : peri-urbanisation, Belgium, Switzerland, Denmark, land policy, mobility, urban planning

Mots-clés : périurbanisation, Belgique, Suisse, Danemark, politique foncière, mobilité, aménagement du territoire

\section{AUTEURS}

JEAN-MARIE HALLEUX

SEGEFA - LEPUR, Université de Liège, Jean-Marie.Halleux@ulg.ac.be

\section{LAURENT BRÜCK}

SEGEFA - LEPUR, Université de Liège, L.Bruck@ulg.ac.be

NICOLAS MAIRY

SEGEFA - LEPUR, Université de Liège, Nicolas.Mairy@ulg.ac.be 